F O U N D T I O N O F C O M P T I N G A D D E C I S I O N S C I E N C S

Vol. 44

(2019)

No. 3

ISSN 0867-6356

sciendo

DOI: $10.2478 /$ fcds-2019-0013

e-ISSN 2300-3405

\title{
Satisficing Newsvendor Problem with the Optimism Coefficient
}

\author{
Milena Bieniek *
}

\begin{abstract}
In this research note the satisficing newsvendor problem is considered which is defined as the maximization of the probability of exceeding the expected profit multiplied by a positive constant. This constant called optimism coefficient can be chosen by the firm's management either based on their preference or the market conditions. The coefficient indicates whether there is a low or high optimistic decision maker. For the general demand distribution the results are significantly dependent on this coefficient.
\end{abstract}

Keywords: inventory control, optimization, newsvendor, optimism coefficient

\section{Introduction}

The newsvendor problem is a fundamental stochastic inventory model $[11,16,19]$. In the classical newsvendor problem the order quantity has to be determined, which maximizes the single period expected profit. Sometimes business companies instead of maximizing the expected profit base decision making on aspiration levels (called also profit targets), where the probability of achieving the aspiration level is maximized $[1,5]$. Simon [17] argues that this is a more sensible approach and it may be better to view profit not as an objective to be maximized, but rather a constraint relative to a given aspiration level. He introduces the term satisficing to this engagement. Later Brown and Sim [2] utilize the concept of aspiration levels to model financial positions, but they additionally mention that their framework is readily applied to other settings. Brown and Sim [2] axiomatize the general class of satisficing measures, which includes success probability measures and also some risk measures, like valueat-risk (VaR). Brown and Sim [2] also state that an important advantage of the satisficing approach is that the aspiration levels are very natural for investors to specify, whereas the traditional models based on risk measures or utility functions

*Economic Faculty, Maria Curie-Sklodowska University, Plac Marii Curie-Sklodowskiej 5, 20-031 Lublin, Poland, milena.bieniek@umcs.lublin.pl 
depend on risk tolerance parameters. Generally risk tolerance parameters are difficult to grasp intuitively for investors and harder to assess appropriately in comparison to assessing aspiration levels. Among many authors, who conclude that the aspiration levels play an important role in the real world, Mao [12] shows in empirical study that managers asked on definition of risk answered that "risk is primary considered to be the prospect of not meeting some target rate of return".

Reference points are used instead of aspiration levels in the reference-dependent theory [10]. However the satisficing approach does not rely on the axioms of utility theory and does not assume that the target has stochastic nature [2]. Another theory connected with satisficing measures is the theory of risk. With regards to this theory Brown and Sim [2] show that probability measures and VaR are dual forms and probability measures are not the special case of VaR. In fact there is a significant difference between them. In the probability measures the aspiration level rather than specifying risk aversion parameters should be fixed. After that the largest value of the aversion parameter is chosen so that the risk of exceeding the target at that parameter is acceptable. Actually the probability measures are highly risk-averse. They explain risk-aversion using the measure of downside risk defined as the critical probability that the target performance level will be obtained. This implies that the firm is only concerned with the risk of not achieving the target and the company is not motivated to overachieve the aspiration level. Such an objective is used if the under-achievement is highly undesirable and the over-achievement is not very rewarding. However, the higher a profit target, the more risk-taking behaviour the target will induce.

One of the first paper devoted to using the probability measures in the newsvendor model is [9]. Later this subject is treated in $[6,13,15]$. In [13] the optimal order rates for normal distribution are given. Recently, in [6] the fixed target profit, which is equal to the maximal expected profit, is studied and in [15] the optimal inventory or capacity allocation are considered. VaR measure is examined by many authors, which is summarized in [4], Chapter 8. Since this is not the scope of this study, a vast research on $\mathrm{VaR}$ is not conducted. Recently risk measures are treated in [3], where the authors create a new measure that incorporates the aspiration-level measure with VaR. For more information on satisficing in newsvendor problem we refer to the book of Choi [4].

In this paper the aspiration level treated in [14] is modified, where the maximization of the probability that the profit will be greater than the expected profit is considered. A new aspiration level is introduced which is defined as the expected profit multiplied by a positive constant $\beta$. The improvement seems to be very simple, but it has a confirmation in real life conditions. It is reasonable to assume that profit targets are not purely preference-dependent but often affected by the opportunities available for the firm [17]. In such cases the firm is able to moderate the aspiration level, depending on its level of optimism influenced by the market circumstances, to be a little lower or higher than the profit they expected. For that reason in this study the constant $\beta$ reflects the level of optimism of the decision maker and is called the optimism coefficient. If $\beta>1$ then it corresponds to a very optimistic decision maker who sets a high profit target to achieve. In case of $\beta \leq 1$ the decision maker is less optimistic and the aspiration level is lower. The interpretation is appropriate if the 
expected profit in the optimal point is non-negative. Otherwise the firm should consider an alternative optimization method. In practice the management is interested more in gaining rather than losing. Although the satisficing objective is ultimate risk-averse regardless of the profit target, as mentioned earlier, the higher optimism coefficient, the more risk-taking behaviour it implies.

The main contribution of this study is that the coefficient $\beta$ has a strong impact on the optimal solution, which is based on a single numerical example. It is worth mentioning that the dependency of the results on the optimism coefficient cannot be described by an elementary mathematical function. It is shown that the case of high optimism is more difficult to consider than other cases and it needs some additional assumptions. The solutions obtained with the optimism coefficient equal to one are equivalent to those obtained by Parlar and Weng [14]. All presented formulas refer to the continuous version of the newsvendor problem.

\section{Existence of non-trivial solution}

Let $p>0$ denote the unit revenue, $c>0$ stand for the unit purchase cost, $s>0$ be the unit shortage cost and $v \in R$ denote the unit salvage value. The salvage value of an item is the value of a remaining item when no further inventory is desired. The salvage value represents the disposal value of the item for a firm. The negative salvage value is called the salvage cost and depends on what is done with the remaining items [7, 14]. The standard assumption is $v<c<p$. The demand is the non-negative random variable $X$ with a known distribution function $F(x)$, the density function $f(x)$ and expected value $E(X)=\mu$. The order quantity $Q$ is the only decision variable in the newsvendor model. If the realized value of the demand is $x$, then the realized profit is given by

$$
\pi(x, Q)= \begin{cases}p x+v(Q-x)-c Q, & \text { if } x \leq Q, \\ p Q-s(x-Q)-c Q, & \text { if } x>Q .\end{cases}
$$

Thus the profit $\pi(X, Q)$ is also random and depends on the order quantity $Q$ and the random demand $X$. It leads to the well known expected profit function $E(Q)=$ $E[\pi(X, Q)]$ given by

$$
E(Q)=(p-v) \mu+(v-c) Q-(p+s-v) \int_{Q}^{\infty}(x-Q) f(x) d x .
$$

The problem is to determine the optimal order quantity $Q$, which obviously depends on the adopted optimality criterion. In the classical solution to the problem the optimal order quantity $Q_{E}^{*}$ is chosen, which maximizes $E(Q)$ and is given by $F\left(Q_{E}^{*}\right)=$ $\frac{p+s-c}{p+s-v}$. It is worth mentioning that although $E(0)=-s \mu$ (the shortage costs must be paid) and $E(\infty)=-\infty$, an assumption is made that $\max E(Q)>0$, i.e. the maximal expected profit is positive.

Alternative optimality criterion proposed in [14], is to maximize the probability $P[\pi(X, Q) \geq E(Q)]$ of exceeding the expected profit. The aspiration level is modified 
and the objective function is defined as the maximization of the success probability given by

$$
P[\pi(X, Q) \geq \beta E(Q)],
$$

where $\beta>0$ is the optimism coefficient. Although, it is a simple modification, it brings some non-trivial results, especially for $\beta>1$. The advantage of that form is that through the use of parameter $\beta$ the aspiration level can be defined in a more general and flexible manner because the optimism coefficient can also be specified by the exogenous market conditions. The disadvantages of this approach is the necessity for some constraints on $Q$ which, for a given $\beta$, ensure that

$$
\beta E(Q) \leq \pi_{\max }(Q)
$$

where

$$
\pi_{\max }(Q)=(p-c) Q
$$

is the maximum profit level and for which the success probability is positive. The condition (4) is equivalent to

$$
R(Q) \leq \frac{p-c}{\beta}
$$

where

$$
R(Q)=\frac{E(Q)}{Q}
$$

and $Q$ is a decision variable.

Theorem 1 A solution to the satisficing-level problem (3) with the optimism coefficient $\beta>0$, which assures that $P(\Pi(Q) \geq \beta E(Q))>0$, exists if

1. $\beta \leq 1$, or

2. $\beta>1$ and

$$
p+s-c-(p+s-v) F\left(Q_{T}^{*}\right) \leq \frac{p-c}{\beta},
$$

where $Q_{T}^{*}$ is the (unique) solution to equation

$$
\int_{Q_{T}^{*}}^{\infty}\left(x-Q_{T}^{*}\right) f(x) d x=\frac{p-v}{p+s-v} \mu-Q_{T}^{*}\left(1-F\left(Q_{T}^{*}\right)\right),
$$

or

3. $\beta>1$ and (8) does not hold and the order quantity belongs to the interval $\left(0, Q_{L}\right) \cup\left(Q_{P}, \infty\right)$, where $Q_{L}$ and $Q_{P}$ are the solutions to the equation

$$
p+s-c-(p+s-v) F(Q)=\frac{p-c}{\beta} .
$$

For the sake of simplicity if $\beta>1$ then further study is done for the model parameters satisfying (8). 


\section{Success probability with the optimism coefficient}

Assuming that the parameters of the model satisfy (8), the success probability $H(Q)=$ $P(\pi(X, Q) \geq \beta E(Q))$ can be written in the form

$$
H(Q)=P\left(D_{1}(Q) \leq X \leq D_{2}(Q)\right)=\int_{D_{1}(Q)}^{D_{2}(Q)} f(x) d x .
$$

The integral limits $D_{1}(Q)$ and $D_{2}(Q)$ are non-negative functions of $Q$ and $\beta$. Using the expressions from [14] the lower limit function $D_{1}(Q)$ is given by $D_{1}(Q)=\max (0, \xi(Q))$, where

$$
\xi(Q)=\frac{\beta E(Q)+(c-v) Q}{p-v} .
$$

If $E(Q) \geq \frac{-(c-v) Q}{\beta}$ then $\xi(Q) \geq 0$, otherwise $\xi(Q)<0$ and consequently $D_{1}(Q)=0$. The upper limit function $D_{2}(Q)$ is given by

$$
D_{2}(Q)=\frac{(p+s-c) Q-\beta E(Q)}{s} .
$$

In order to analyse the variability of $H(Q)$ the variability of the limit functions is investigated first. The analysis is similar to those given in [14], however $\beta$ cannot be introduced automatically and the dependence on $\beta$ is not described by any elementary function. Note that $\xi(0)=-\frac{\beta s}{p-v} \mu<0$,

$$
\lim _{Q \rightarrow \infty} \xi(Q)= \begin{cases}\mu, & \text { if } \beta=1, \\ +\infty, & \text { if } 0<\beta<1, \\ -\infty, & \text { if } \beta>1,\end{cases}
$$

$\xi^{\prime}(Q)=\frac{\beta(p+s-v)}{p-v}(1-F(Q))-\frac{(\beta-1)(c-v)}{p-v}$ and $\xi^{\prime \prime}(Q)=-\frac{\beta(p+s-v)}{p-v} f(Q)<0$. Moreover, $\xi^{\prime}(Q)>0$ for $Q \geq 0$ and $\beta \leq 1$ and it can be stated that $\xi(Q)$ has a unique zero at $Q_{0}$. Then $D_{1}(Q)$ is equal to 0 up to $Q_{0}$, and then it is an increasing function of $Q$. Next, for $\beta>1$, the equation $\xi^{\prime}(Q)=0$, or equivalently

$$
F(Q)=1-\left(1-\frac{1}{\beta}\right) \frac{c-v}{p+s-v} .
$$

has the unique root $Q_{1}$. Therefore the function $\xi$ is increasing on $\left(0, Q_{1}\right)$ from $-\frac{\beta s \mu}{p-v}$ to $\xi\left(Q_{1}\right)$ and then decreasing on $\left(Q_{1}, \infty\right)$ to $-\infty$. In order to prove that $\xi\left(Q_{1}\right)>0$ the number of zeros of $\xi(Q)$ is studied. Note that $\xi(Q)=0$ if and only if

$$
R(Q)=\frac{v-c}{\beta},
$$

where $R(Q)$ is defined by (7). For $\beta>1$ the last quantity is negative but greater than $R(\infty)=v-c$. Since $R\left(Q_{T}^{*}\right)>0$ then taking into account monotonicity of $R(Q)$ it is observed that (15) has exactly two solutions, and $\xi$ has exactly two zeros $Q_{0}$ and 


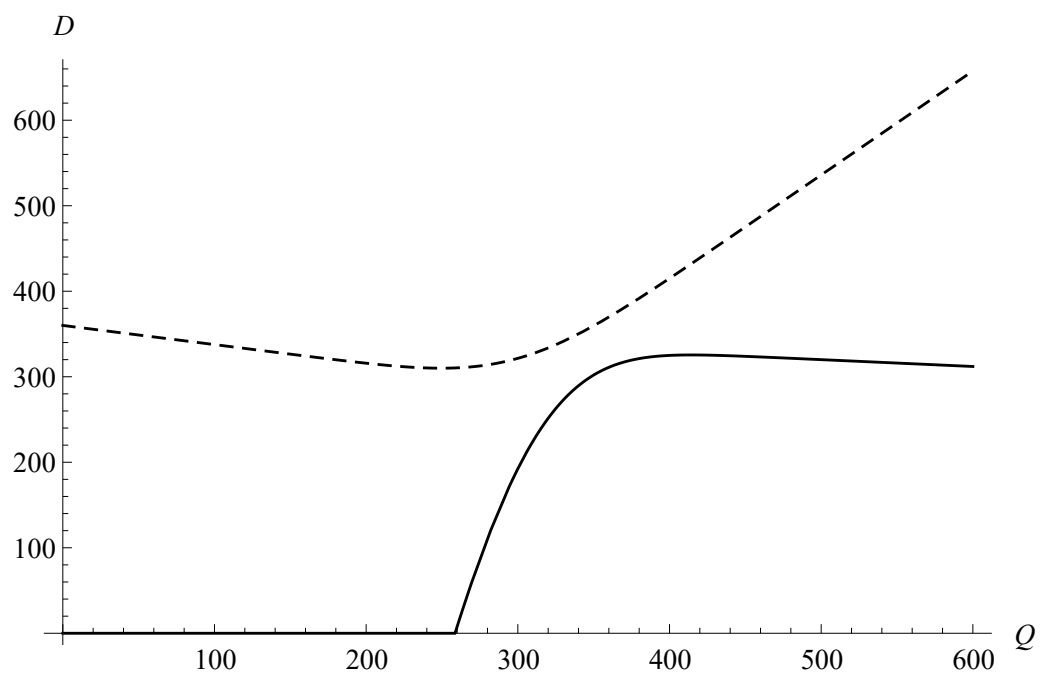

Figure 1. Limit functions $D_{1}(Q)$ (solid) and $D_{2}(Q)$ (dashed) for normal distribution and model parameters $(\mu, \sigma, v, c, p, s)=(300,50,15,19,25,50)$ with $\beta=1.2$.

$Q_{0}^{\prime}$. Since $D_{1}(Q)=\max (0, \xi(Q))$, then $D_{1}(Q)$ is equal to 0 on $\left(0, Q_{0}\right)$, increasing on $\left(Q_{0}, Q_{1}\right)$, decreasing on $\left(Q_{1}, Q_{0}^{\prime}\right)$ and equal to 0 for $Q \geq Q_{0}^{\prime}$.

It is noted that $D_{2}(0)=\beta \mu>0, D_{2}^{\prime}(Q)=\frac{(1-\beta)(p+s-c)}{s}+\frac{\beta(p+s-v)}{s} F(Q)$ and for $\beta<1$ and all $Q \geq 0$ the derivative $D_{2}^{\prime}(Q)>0$. Therefore the function $D_{2}(Q)$ tends to infinity as $Q \rightarrow \infty$. On the other hand, for $\beta>1$ the derivative $D_{2}^{\prime}(Q)=0$ for some $Q_{2}$ satisfying the equality

$$
F\left(Q_{2}\right)=\left(1-\frac{1}{\beta}\right) \frac{p+s-c}{p+s-v} .
$$

Moreover, since the second derivative $D_{2}^{\prime \prime}(Q)=\beta \frac{p+s-v}{s} f(Q)$ is positive, then the function $D_{2}(Q)$ is convex. The limit function $D_{2}(Q)$ is decreasing up to $Q_{2}$, then tends to infinity for $Q>Q_{2}$ and attains the minimum at the point $Q_{2}$, which is well-defined since $0 \leq F\left(Q_{2}\right) \leq 1$. The graphs of the functions $D_{2}(Q)$ and $D_{1}(Q)$ for $\beta=1.2$ are plotted in Figure 1 .

Now the analysis of the variability of the difference $D_{2}(Q)-D_{1}(Q)$ is necessary. Since (6) should be satisfied then $D_{2}(Q)-D_{1}(Q)=\frac{p+s-v}{s(p-v)}((p-c) Q-\beta E(Q)) \geq 0$. The point at which $D_{2}(Q)-D_{1}(Q)$ reaches the minimum is specified below.

Lemma 1 For $\beta>1$ the difference $D_{2}(Q)-D_{1}(Q)$ is minimized at the unique point $Q_{M}$ given by

$$
F\left(Q_{M}\right)=\frac{s}{p+s-v}+\left(1-\frac{1}{\beta}\right) \frac{p-c}{p+s-v} .
$$

For $\beta \leq 1$ if

$$
s+\left(1-\frac{1}{\beta}\right)(p-c)>0
$$


Table 1. The values of $H\left(Q_{H}^{*}\right)$ and $Q_{H}^{*}$ for normal distribution and the model parameters $(\mu, \sigma, v, c, p, s)=(300,50,15,19,25,50)$.

\begin{tabular}{|c|c|c|c|c|c|}
\hline$\beta$ & 0.8 & 0.9 & 1.0 & 1.1 & 1.2 \\
\hline$Q_{H}^{*}$ & 336.24 & 323.57 & 314.52 & 307.2 & 302.44 \\
\hline$H\left(Q_{H}^{*}\right)$ & 0.778 & 0.732 & 0.697 & 0.671 & 0.652 \\
\hline
\end{tabular}

then $D_{2}(Q)-D_{1}(Q)$ is minimized at the unique point $Q_{M}$ given by (17), otherwise $D_{2}(Q)-D_{1}(Q)$ is an increasing function of $Q$ for all $Q \geq 0$.

Remark 1 In order to assure (8), the solution in the interval $\left(Q_{2}, Q_{1}\right)$ is considered. Then $Q_{2} \leq Q_{M} \leq Q_{1}$. Note also that $Q_{M}<Q_{E}^{*}$, where $Q_{E}^{*}$ is the point for which $E(Q)$ is maximal.

Now the properties of $H(Q)$ defined by (11) are analysed. It should be emphasized, that in general $Q_{M}$ which minimizes $D_{2}(Q)-D_{1}(Q)$ does not have to minimize $H(Q)$. The set of acceptable assumptions allowing the study of variability of the success probability as a function of $Q$ is given.

Theorem 2 1. If $\beta>1$, the model parameters assure that $Q_{2}<Q_{0}$ and the demand density satisfies

$$
a(Q)<b(Q) \text { for all } Q_{M}<Q<Q_{1},
$$

where $a(Q)=\frac{f\left(D_{2}(Q)\right)}{f\left(D_{1}(Q)\right)}$ and $b(Q)=\frac{s}{p-v} \frac{\beta(p+s-v)(1-F(Q))+(1-\beta)(c-v)}{\beta(p+s-v) F(Q)+(1-\beta)(c-v)}$, then $H(Q)$ has a local maximum on $\left(Q_{2}, Q_{1}\right)$ at some $Q_{H}^{*} \in\left[Q_{0}, Q_{M}\right]$.

2. If $\beta \leq 1$ and the demand density satisfies (19) with $Q_{1}=\infty$, then $H(Q)$ is maximized at some $Q_{H}^{*} \in\left[Q_{0}, Q_{M}\right]$.

Remark 2 It is possible that $E\left(Q_{H}^{*}\right)<0$. In this case all theorems hold true but the notion of the optimism coefficient looses its meaning. The company should revise its policy and consider an alternative optimization method.

Since it is hard to examine analytically the dependence of the results on $\beta$, a numerical example is given. In Table 1 numerically obtained values of $H(Q)$ and the corresponding order quantities are presented for various values of $\beta>0$. The newsvendor problem with parameters $(\mu, \sigma, v, c, p, s)=(300,50,15,19,25,50)$ and normal demand distribution $N(\mu, \sigma)$ is considered. The parameters attain the same values as those treated in [14]. Note that as $\beta$ increases from 0.8 to 1.2 with step 0.1 , the order quantity $Q_{H}^{*}$ decreases from 336.24 to 302.44 and $H\left(Q_{H}^{*}\right)$ decreases from 0.778 to 0.652 . In Figure 2 the variability of $H(Q)$ is presented for $\beta=0.9 ; 1.0 ; 1.1$, respectively. This implies that the optimism coefficient plays an important role on the maximization of the success probability. In presented example $Q_{H}^{*}$ and $H\left(Q_{H}^{*}\right)$ for higher values of $\beta$ are lower. 


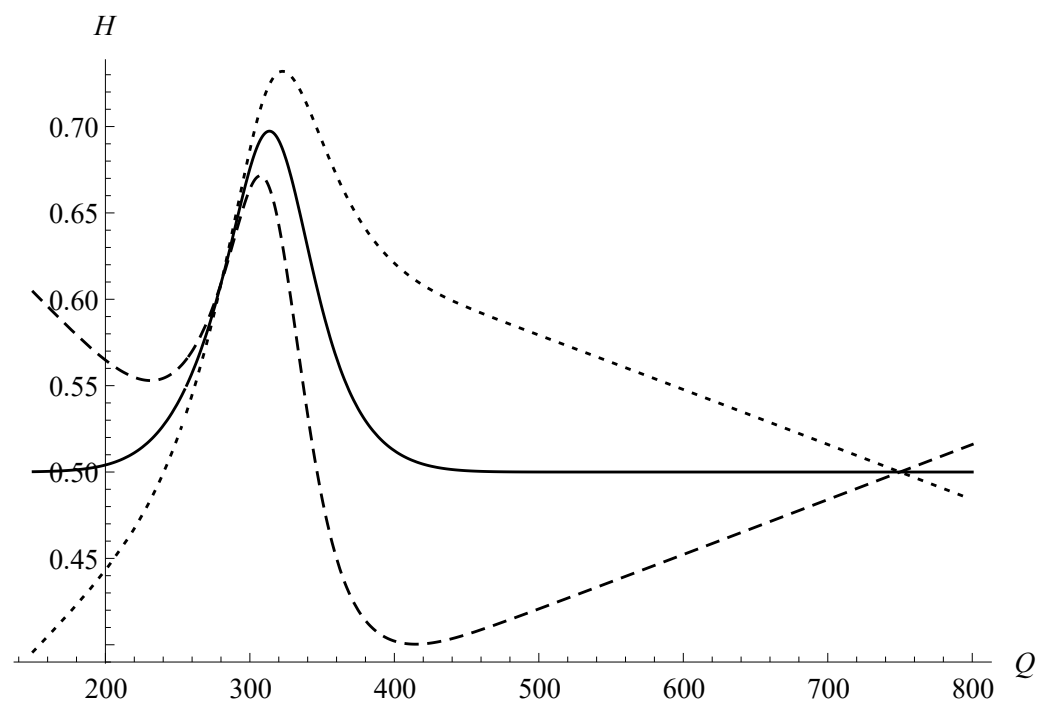

Figure 2. $H(Q)$ for normal distribution and model parameters $(\mu, \sigma, v, c, p, s)=$ $(300,50,15,19,25,50)$ with $\beta=0.9$ (dotted); $\beta=1.0$ (solid) and $\beta=1.1$ (dashed).

\section{Conclusions}

In this paper the results of [14] concerning the solution to satisficing-level newsvendor problem have been extended . The aspiration level studied in the mentioned paper is modified by introducing the optimism coefficient. This is a positive constant, which can be fixed by the management of the firm taking into account internal (decision maker optimism) and external (market climate) conditions. If the optimism coefficient is less or equal to one, a newsvendor is less optimistic and market conditions are unfavourable. The optimism coefficient greater than one assumes a very optimistic newsvendor and favorable market circumstances.

The described problem is solved for any positive value of optimism coefficient. The newsvendor model with high optimism coefficient is more demanding and consequently different than models for other values. Therefore in this case some necessary assumptions on the order quantity should be made and the derivations could not be immediately obtained from the results of [14]. The main result of this paper is proving that the constant introduced in the aspiration level significantly influences the solution in a non straightforward way. The given probability measure may be useful in practice since most firms prefer assigning target profits to estimating risk parameters and simple solutions are more likely to be used in the real world. 


\section{Appendix}

Proof of Theorem 1

If $\beta \in(0,1]$ then the condition (6) is satisfied for any $Q \geq 0$. However, for given $\beta>1$ it is possible that (6) fails. Now the monotonicity of $R(Q)$ given by (7) is studied. Since $E(0)=-s \mu<0$ then $R(0)=\lim _{Q \rightarrow 0} \frac{E(Q)}{Q}=-\infty$. Moreover since

$$
E^{\prime}(Q)=v-c+(p+s-v)(1-F(Q))
$$

then $\lim _{Q \rightarrow \infty} E^{\prime}(Q)=v-c$ and by de l'Hospital rule $R(\infty)=\lim _{Q \rightarrow \infty} E^{\prime}(Q)=$ $v-c<0$. The derivative of $R(Q)$ is equal to $R^{\prime}(Q)=\frac{Q E^{\prime}(Q)-E(Q)}{Q^{2}}$. Now using the expressions (2) and (20) the result is

$$
R^{\prime}(Q)=\frac{1}{Q^{2}}\left((p+s-v) \int_{Q}^{\infty} x f(x) d x-(p-v) \mu\right)=\frac{1}{Q^{2}} T(Q) .
$$

Note that $T^{\prime}(Q)=-(p+s-v) Q f(Q)<0, \quad T(0)=s \mu>0$, and $T(\infty)=-(p-$ $v) \mu<0$. Hence $T(Q)=0$ for the unique $Q$, denoted by $Q_{T}^{*}$. Therefore the function $T(Q)$ is first positive, then negative, and consequently $R(Q)$ is first increasing and then decreasing. Accordingly $R(Q)$ has the unique maximum for $Q=Q_{T}^{*}$ and since $\max E(Q)>0$, then $R\left(Q_{T}^{*}\right)>0$. By (21) the quantity $Q_{T}^{*}$ is the (unique) solution to equation (9) or equivalently $R\left(Q_{T}^{*}\right)=v-c+(p+s-v)\left(1-F\left(Q_{T}^{*}\right)\right)$. Since $R\left(Q_{T}^{*}\right)$ is positive, then by (6) the parameters $p, s, c$ and $v$ of the newsvendor model should satisfy (8). If (8) does not hold, then the order quantity should belong to the intervals $\left(0, Q_{L}\right) \cup\left(Q_{P}, \infty\right)$, where $Q_{L}$ and $Q_{P}$ are the solutions to the equation (10).

Proof of Lemma 1

First the case $\beta>1$ is considered. Since

$$
D_{2}^{\prime}(Q)-D_{1}^{\prime}(Q)=\frac{p+s-v}{s(p-v)}[(1-\beta)(p-c)-\beta s+\beta(p+s-v) F(Q)]
$$

then (17) can be obtained from the equality $D_{2}^{\prime}\left(Q_{M}\right)-D_{1}^{\prime}\left(Q_{M}\right)=0$. Moreover, the second derivative $D_{2}^{\prime \prime}(Q)-D_{1}^{\prime \prime}(Q)=\frac{\beta(p+s-v)^{2}}{s(p-v)}$ is positive for all $Q \geq 0$. Therefore, the difference $D_{2}(Q)-D_{1}(Q)$ is a convex function of $Q$ and attains its minimum value at $Q_{M}$. Note that for $\beta>1$ the point $Q_{M}$ is well-defined because $0 \leq F\left(Q_{M}\right) \leq 1$. For $\beta \leq 1$ the existence of $Q_{M}$ follows from (18).

Proof of Theorem 2

Note that if $\beta>1$ and $Q_{2}<Q_{0}$ then $D_{1}^{\prime}(Q)=0$ and $D_{2}^{\prime}(Q)>0$. Consequently, $H(Q)$ is increasing on $\left(Q_{2}, Q_{0}\right)$ and the demand density is positive except for possibly finite number of points. Furthermore $H^{\prime}(Q)=f\left(D_{2}(Q)\right) D_{2}^{\prime}(Q)-f\left(D_{1}(Q)\right) D_{1}^{\prime}(Q)$ and the condition assuring the negativity of $H^{\prime}(Q)$ for $Q \geq Q_{M}$ follows from (19). The proof is similar if $\beta \leq 1$. 


\section{Acknowledgment}

This work was supported by grant of National Science Centre, Poland, no 2018/02/X/HS4/00167.

\section{References}

[1] Barbati M., Greco S., Kadziński M., Słowiński R., Optimization of multiple satisfaction levels in portfolio decision analysis, Omega, 78, 2018, 192-204.

[2] Brown D. B., Sim M., Satisficing Measures for Analysis of Risky Positions, Management Science, 55, 2009, 71-84.

[3] Chen, Gongtao L., Long D.Z., Perakis G., The Impact of a Target on Newsvendor Decisions, Manufacturing $\&$ Service Operations Management, 17, 2015, 78-86.

[4] Choi T.-M., ed. Handbook of newsvendor problems, Int Ser in Oper Res Manag Sci, New York: Springer Science+Business Media, 341-359, 2012.

[5] Halpern J.J., Stern R.N., Debating rationality: nonrational aspects of organizational decision making, Frank W. Pierce memorial lectureship and conference series, Ithaca, N.Y.: ILR Press, 1998.

[6] He X., Khouja M., Pareto analysis of supply chain contracts under satisficing objectives, European Journal of Operational Research, 214, 2011, 53-66.

[7] Hiller F.S., Lieberman G.J., Introduction to Operations Research, Boston, 7th ed. McGraw-Hill Series in Engineering and Management Science, 2001.

[8] Jardón C.M., Satisfaction level and competitiveness in subsistence small businesses, Management Decision, 56, 5, 2018, 1108-1121.

[9] Kabak I.W., Shiff A.I., Inventory models and management objectives, Sloan Management Review, 19, 1978, 53-59.

[10] Kahneman D., Tversky A., Prospect Theory: An Analysis of Decision under Risk, Econometrica, 47, 1979, 263-291.

[11] Khouja M., The single-period (news-vendor) problem: literature review and suggestions for future research, Omega, 27, 1999, 537-553.

[12] Mao J.C.T., Survey of capital budgeting: theory and practice Journal of Finance, 25, 1970, 349-360.

[13] Norland R., Refinements in Ismail-Loderback's stochastic CVP model, Decision Sciences, 11, 1980, 562-572.

[14] Parlar M., Weng Z.K., Balancing desirable but conflicting objectives in the newsvendor problem, IIE Transactions, 35, 2003, 131-142. 
[15] Pinto R., Stock rationing under a profit satisficing objective, Omega, 65, 2016, 55-68.

[16] Qin Y., Wang R., Vakharia A.J., Chen Y., The newsvendor problem: review and directions for future research, European Journal of Operational Research, 213, 2011, 361-374.

[17] Simon H.A., A behavioral model of rational choice, Quartely Journal of Economics, 69, 1955, 99-118.

[18] Simon H.A., Theories of decision-making in economics and behavioral science, Amererican Economic Review, 49, 1959, 253-283.

[19] Zipkin P.H., Foundations of Inventory Management, Boston, McGraw-Hill, 2000.

Received 04.10.2018, Accepted 09.04.2019 